\title{
Research on the effect of different test cycles on low temperature emissions and fuel consumption of Light plug-in hybrid cars
}

\author{
Fan Yang*, Chongzhi Zhong, and Chun Li \\ China Automotive Technology And Research Center
}

Keywords: plug-in hybrid, low temperature emissions, fuel consumption, WLTC cycle, CLTC-P cycle.

\begin{abstract}
Based on a plug-in hybrid car equipped with a $1.5 \mathrm{~L}$ turbocharged direct injection gasoline engine, this paper studies the emission and fuel consumption characteristics of the prototype vehicle under three different cycle conditions, WLTC city, WLTC and CLTC-P. The results show that for the four pollutants: CO, THC, NMHC and NOx, WLTC city cycle emissions are the largest, WLTC cycle emissions are the smallest, CLTC-P cycle emissions are the middle. For $\mathrm{N}_{2} \mathrm{O}$, WLTC cycle has the largest combined emissions, CLTC-P cycle combined emissions are the smallest, and WLTC city cycle combined emissions are the center. The combined fuel consumption under WLTC city conditions is approximately 1.3 times the combined fuel consumption under the complete WLTC cycle. The combined fuel consumption under CLTC-P conditions is approximately 1.25 times that of the complete WLTC cycle. The first phase of each cycle is the phase with the highest emissions and fuel consumption.
\end{abstract}

\begin{tabular}{lc}
\multicolumn{2}{l}{ Abbreviations } \\
WLTC & World light vehicle unified test cycle \\
WLTC city & Low and medium speed phases of WLTC \\
CLTC-P & China light-duty vehicle test cycle for \\
& passenger car \\
CO & Carbon monoxide \\
THC & Total hydrocarbons \\
NMHC & Non-methane hydrocarbons \\
NOx & Nitrogen oxides \\
$\mathrm{N}_{2} \mathrm{O}$ & Nitrous oxide
\end{tabular}

\section{Introduction}

\footnotetext{
*Corresponding author: yangfan2017@catarc.ac.cn
} 
With the increase of global car ownership in recent years, the greenhouse effect is becoming more and more severe. According to statistics, there are more than 700 million cars in the world in the $21 \mathrm{st}$ century [1-2]. At the current rate of increase in $\mathrm{CO}_{2}$ concentrations, atmospheric $\mathrm{CO}_{2}$ levels would double by 2100.The United Nations assessment reports that global average temperatures would then rise by $1.0 \sim 3.5^{\circ} \mathrm{C}$ than they are now, causing extreme ice to melt and sea levels to rise by 15 95 centimetres, thus flooding large economically developed coastal areas and possibly a series of other serious problems [3]. Governments around the world have begun to focus on this situation and its harmful consequences and to discuss the issue of reducing $\mathrm{CO}_{2}$ emissions [4-5].

In China, The Ministry of Industry and Information Technology, the Ministry of Finance, the Ministry of Commerce, the General Administration of Customs and the General Administration of Quality Supervision and Inspection issued the "measures for the parallel management of the average fuel consumption of passenger car enterprises and the points of new energy vehicles" in 2017. By 2020, the average fuel consumption of passenger cars produced in that year fell to $5.0 \mathrm{~L} / 100 \mathrm{~km}$, and the fuel consumption of energy-efficient passenger cars fell below $4.5 \mathrm{~L} / 100 \mathrm{~km}$ [6]. It can be seen that the management of vehicle energy saving and emission reduction is becoming more and more strict.

Plug-in hybrid electric vehicle is a kind of vehicle type between pure electric vehicle and traditional fuel vehicle, which can drive higher mileage in the state of only consuming electric energy, because it has internal combustion engine and can provide power by burning fuel when the electric energy is insufficient, thus it has the advantage of more flexible use than pure electric vehicle [7-9]. Compared with fossil-fuel-based internal combustion engines, electric energy is generally considered to be cleaner and more efficient, and the actual driving distance of most car users per day is less than $80 \mathrm{~km}$, and the pure electric range of plug-in hybrid vehicles can exactly meet this requirement [10-11].

This paper takes a light plug-in hybrid vehicle with $1.5 \mathrm{~L}$ turbocharged direct injection gasoline engine as the research object carries on the low temperature emission and the fuel consumption test of the vehicle electric quantity holding mode under the WLTC city, WLTC and CLTC-P three working conditions cycle, and measures the CO, THC, NMHC, $\mathrm{NOx}, \mathrm{N}_{2} \mathrm{O}$ and the $\mathrm{CO}_{2}$ emission of the plug-in hybrid car. The pollutants and fuel consumption under three different measuring conditions were compared and analyzed by the control variable method.

\section{Test conditions and test vehicle and equipment}

\subsection{Introduction of different test cycles}

\subsubsection{WLTC test cycle and WITC city test cycle}

WLTC test cycle is a four-part variable operating conditions test cycle, which is divided into low-speed section, medium-speed section, high-speed section and extra-high-speed section, in which low-speed section duration and time $589 \mathrm{~s}$, medium-speed segment duration 433s, high-speed segment duration 455s, extra-high-speed segment duration $323 \mathrm{~s}$, total operating time $1800 \mathrm{~s}$, total distance $23.27 \mathrm{~km}[12]$. The WLTC city test cycle is the first two parts of the WLTC: the low speed section and the middle speed section, which are used to measure the standard test cycle of the exhaust pollutants in the low temperature and cold start of the Chinese Six phase vehicles. 


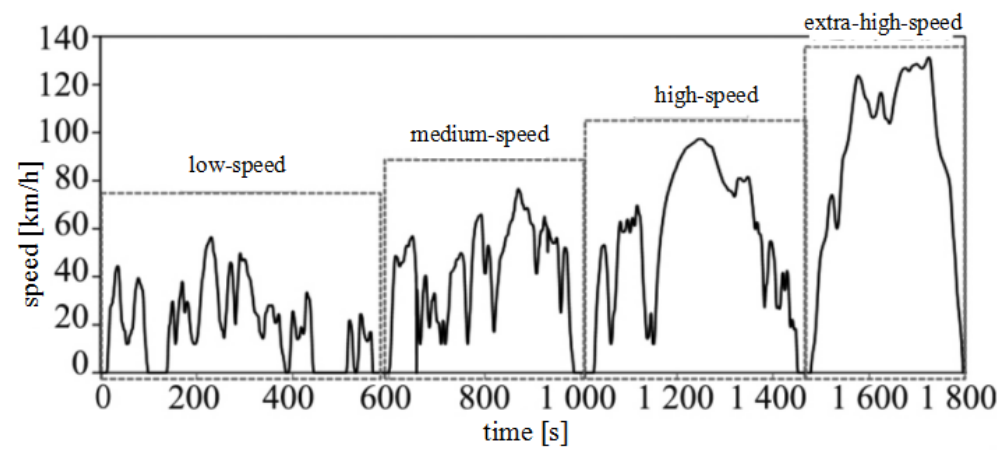

Fig. 1. WLTC Velocity curve of cycle condition.

\subsubsection{CLTC-P test cycle}

Chinese passenger car driving condition (CLTC-P) include low speed (1 part), medium speed ( 2 parts) and high speed ( 3 parts) three speed intervals, the whole CLTC-P test cycle conditions total 1800 seconds [13], the operating conditions curve is shown in the diagram:

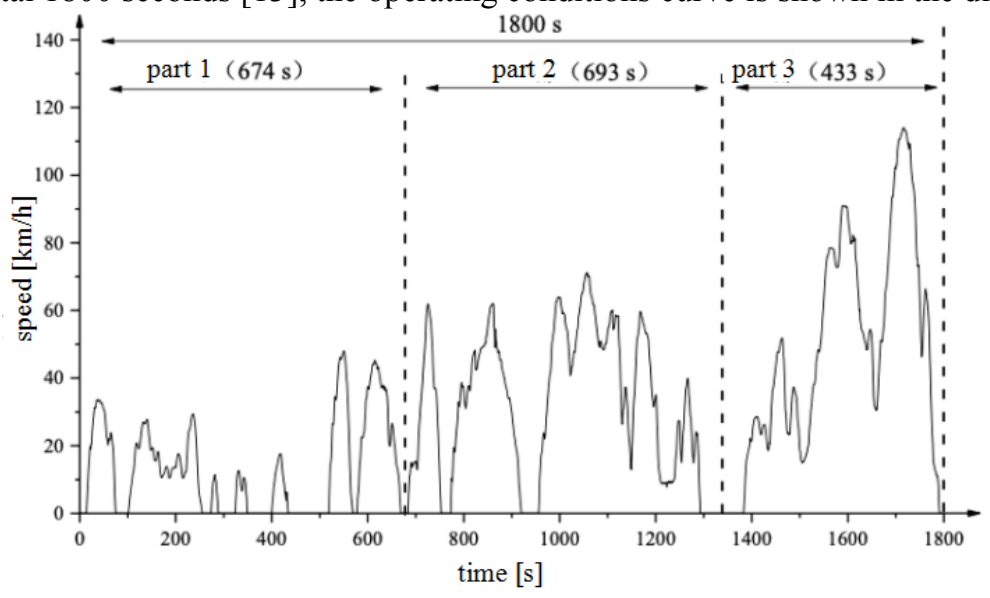

Fig. 2. CLTC-P Velocity curve of cycle condition.

\subsubsection{Comparison of characteristic parameters of two test cycles}

WLTC test cycle and CLTC test cycle are variable condition test cycle,their total duration is the same as 1800 seconds, but there are differences in total cycle mileage and maximum speed.The characteristic parameters of the two working conditions are shown in the table1: 


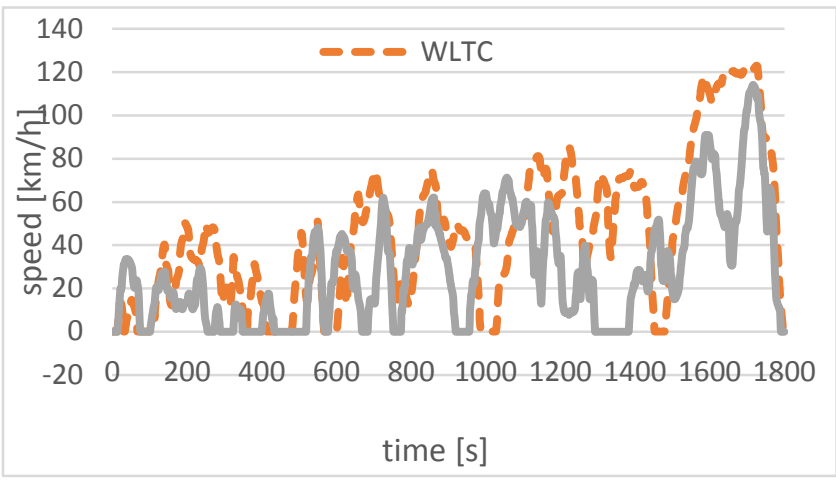

Fig. 3. Comparison of two test cycles.

Table1. Comparison of characteristic parameters of WLTC and CLTC-P cycles.

\begin{tabular}{|l|c|c|c|c|c|c|c|}
\hline \multirow{2}{*}{ parameters } & \multicolumn{2}{|l|}{ CLTC-P cycle } & \multicolumn{2}{l}{ WLTC cycle } \\
\cline { 2 - 8 } & Part1 & Part2 & Part3 & Low & Middle & High & $\begin{array}{c}\text { Extra-h } \\
\text { igh }\end{array}$ \\
\hline period(s) & 674 & 693 & 433 & 588 & 432 & 454 & 322 \\
\hline distance(km) & 2.45 & 5.91 & 6.12 & 3.09 & 4.72 & 7.12 & 8.25 \\
\hline $\begin{array}{l}\text { Max speed } \\
\text { (km/h) }\end{array}$ & 48.1 & 71.2 & 114 & 56.5 & 76.6 & 87.4 & 131.3 \\
\hline $\begin{array}{l}\text { Max acceleration } \\
\text { (m/s })\end{array}$ & 1.47 & 1.44 & 1.06 & 1.61 & 1.39 & 1.67 & 1.06 \\
\hline $\begin{array}{l}\text { Max deceleration } \\
\text { (m/s })\end{array}$ & -1.42 & -1.47 & -1.46 & -1.5 & -1.5 & -1.5 & -1.44 \\
\hline $\begin{array}{l}\text { average speed } \\
\text { (km/h) }\end{array}$ & 13.09 & 30.68 & 50.9 & 18.91 & 39.25 & 56.37 & 92 \\
\hline $\begin{array}{l}\text { average acceleration } \\
\text { (m/s2) }\end{array}$ & 0.42 & 0.46 & 0.46 & 0.48 & 0.42 & 0.34 & 0.3 \\
\hline $\begin{array}{l}\text { average deceleration } \\
\text { (m/s2) }\end{array}$ & -0.45 & -0.5 & -0.54 & -0.44 & -0.57 & -0.43 & -0.34 \\
\hline $\begin{array}{l}\text { Acceleration time } \\
\text { ratio(\%) }\end{array}$ & 22.55 & 30.45 & 35.8 & 34.63 & 49.65 & 49.02 & 50.77 \\
\hline $\begin{array}{l}\text { Deceleration time } \\
\text { ratio(\%) }\end{array}$ & 21.51 & 28.43 & 30.95 & 37.69 & 36.49 & 38.46 & 45.2 \\
\hline Constant Time Ratio(\%) & 20.77 & 21.36 & 27.71 & 2.38 & 2.77 & 5.93 & 1.86 \\
\hline Idle Time Ratio(\%) & 35.16 & 19.77 & 5.54 & 25.3 & 11.09 & 6.59 & 2.17 \\
\hline
\end{tabular}

\subsection{Test vehicle and test equipment}

The main technical parameters of the vehicle under test are shown in Table2. The fuel used is China national six standard 95\# benchmark gasoline.

The environmental warehouse used for low temperature test is the SD 520'/30 type environmental warehouse produced by German WEISS Company, the Chassis dynamometer is the RPL1220 type single axis chassis dynamometer produced by Austrian AVL Company, and the emission analysis system is the MEXA-ONE type light vehicle emission analysis system produced by Japanese HORIBA Company. The measurement of THC is hydrogen flame ion method, $\mathrm{CO}$ by non-dividing infrared method, NOx by chemiluminescence method, and $\mathrm{N}_{2} \mathrm{O}$ by infrared absorption method. 
The principle of hydrogen flame ion method (FID) is that hydrocarbons can be ionized into electrons and free ions when they burn at about $2000^{\circ} \mathrm{C}$ of high temperature of hydrogen flame, and the number of ions is basically proportional to the number of carbon atoms. The ions decomposed in the hydrogen flame form electron flow under the voltage between the plates of the ion absorption electrode, the current size represents the concentration of carbon atoms in the sample gas, so the result of FID detection is the ppm value of carbon atoms in the sample gas.

Table 2. Main technical parameters of test vehicle.

\begin{tabular}{cc}
\hline Project & Technical Parameter \\
\hline Engine & $\begin{array}{c}\text { Direct injection\& } \\
\text { turbocharging } \\
\text { paralleling }\end{array}$ \\
Hybrid mode & $1.5 \mathrm{~L}$ \\
Engine displacement & $130 \mathrm{~kW}$ \\
Engine rating power & $110 \mathrm{~kW}$ \\
Motor rating power & Front-wheel driven \\
Drive mode & DCT \\
Transmission form &
\end{tabular}

The non-dispersive infrared analysis (NDIR) is the best method for the determination of $\mathrm{CO}$ at present. Its working principle is based on measuring the energy absorption of gas to infrared rays at a specific wavelength. CO can absorb the wavelength of $4.5 \sim 5.0 \mu \mathrm{m}$ infrared ray, with absorption peak, the concentration of $\mathrm{CO}$ in the sample gas can be obtained by infrared ray through a certain length of the transmission energy of the gas. In order to reduce the interference of other gases, a filter chamber is set up in front of the sample chamber to filter out the wavelength corresponding to the other interference gases.

The chemiluminescence method (CLD) used to analyze the NOx,CLD can only determine NO directly. Sample gas and excess ozone are mixed and chemically reacted in the reaction chamber to form $\mathrm{NO}_{2}$, about $10 \%$ of which are in the electronically excited state. When the $\mathrm{NO}_{2}$ of the excited state attenuates to the ground state, the emission wavelength is $0.6 \sim 3 \mu \mathrm{m}$ photons. The chemiluminescence intensity is proportional to the product of NO and ozone concentration and is also related to the measurement conditions. However, when the measurement conditions are constant and the ozone concentration is constant and much higher than the NO concentration. The measurement NOx is actually the sum of the measured $\mathrm{NO}$ and $\mathrm{NO}_{2}$, so in the measurement $\mathrm{The}_{\mathrm{NO}}$ in the exhaust must be converted into NO. before the quantity is measured.

\subsection{Test methods}

\subsubsection{Vehicle pretreatment and test preparation}

Prior to pretreatment, check whether the tire pressure of the vehicle meets the technical requirements, whether the engine oil level is normal, check whether the fuel in the vehicle tank meets the requirements, and preheat the chassis dynamometer at a speed of $100 \mathrm{~km} / \mathrm{h}$ for $20 \mathrm{~min}$. Drive the test vehicle or push it to the dynamometer for fixing and pretest the vehicle according to the WLTC cycle. When the pretest is finished, according to the method of determining the driving resistance CC the GB18352.6-2016 attachment, the vehicle coastdown is carried out according to the test quality and the road load coefficient F0,F1,F2, and the setting parameters of the chassis dynamometer are obtained,and then 
follow WLTC two cycles to preprocess [14]. the WLTC cycle pretreatment here is carried out twice to ensure that the vehicle is in the power holding mode during the vehicle test (that is, the battery SOC is in equilibrium). the vehicle will be pretreated according to the two cycles before each low temperature test later to ensure that the prototype battery SOC is at the same level before each test. When pre-treatment is completed, the vehicle shall be placed in an environmental warehouse for cryogenic immersion, and the prototype shall be placed not less than $12 \mathrm{~h}$ but not more than $36 \mathrm{~h}$.. the average ambient temperature (dry ball) shall be maintained during this period: within $-7^{\circ} \mathrm{C} \pm 3^{\circ} \mathrm{C}$ per hour.

\subsubsection{Prototype test}

The chassis dynamometer and emission analyzer are preheated before the start of the test, and the zero and span points of the emission analyzer are calibrated after preheating. push the test vehicle onto the chassis dynamometer for fixing, check whether the oil temperature and coolant temperature of the test vehicle are in the range of $-7 \pm 3{ }^{\circ} \mathrm{C}$, check the tire pressure of the vehicle and the SOC of the battery to confirm that the status of the prototype is consistent before each low temperature test [15-16]. The final,start the test and run test cycle, during the test, the heating and defrosting device should be closed, etc., the driver must not see the speed tolerance. Maximum tolerance, $2.0 \mathrm{~km} / \mathrm{h}$, time \pm 1.0 seconds inner; tolerance lower limit,$-2.0 \mathrm{~km} / \mathrm{h}$, time within \pm 1.0 seconds. The allowable speed tolerance is greater than the specified requirement, but the excess time can not exceed 1 second. During the test, there should be no more than 10 times of excess speed. During the test, use the tracking fan to cool the engine, the fan should be within $300 \mathrm{~mm}$ in front of the car. After the test, the sampling results were analyzed by reading bag, and the final low temperature emission and fuel consumption results were obtained.

\section{Test results and analysis}

The test results obtained by conducting low temperature tests under three different cycles of WLTC city, WLTC and CLTC-P are shown in the table.

Table 3. Raw data on low temperature emission and fuel consumption under three test cycles

\begin{tabular}{|c|c|c|c|c|c|c|r|}
\hline Test cycle & $\begin{array}{c}\mathrm{CO} \\
\mathrm{g} / \mathrm{km}\end{array}$ & $\begin{array}{c}\mathrm{THC} \\
\mathrm{g} / \mathrm{km}\end{array}$ & $\begin{array}{c}\mathrm{NMHC} \\
\mathrm{g} / \mathrm{km}\end{array}$ & $\begin{array}{c}\mathrm{NOx} \\
\mathrm{g} / \mathrm{km}\end{array}$ & $\begin{array}{c}\mathrm{N}_{2} \mathrm{O} \\
\mathrm{mg} / \mathrm{km}\end{array}$ & $\begin{array}{c}\mathrm{CO}_{2} \\
\mathrm{~g} / \mathrm{km}\end{array}$ & $\begin{array}{c}\text { F.E. } \\
\text { L/100km }\end{array}$ \\
\hline WLTC city & 2.015 & 0.333 & 0.310 & 0.050 & 0.001 & 270.815 & 11.733 \\
\hline WLTC city 1P & 4.949 & 0.852 & 0.793 & 0.118 & 0.002 & 362.446 & 15.865 \\
\hline WLTC city 2P & 0.141 & 0.002 & 0.002 & 0.006 & 0.000 & 212.302 & 9.090 \\
\hline WLTC & 0.690 & 0.111 & 0.102 & 0.018 & 1.048 & 210.390 & 9.060 \\
\hline WLTC 1P & 4.452 & 0.798 & 0.744 & 0.112 & 2.361 & 366.920 & 16.101 \\
\hline WLTC 2P & 0.058 & 0.003 & 0.002 & 0.007 & 1.698 & 239.780 & 10.256 \\
\hline WLTC 3P & 0.129 & 0.004 & 0.003 & 0.004 & 1.114 & 161.310 & 6.909 \\
\hline WLTC 4P & 0.132 & 0.009 & 0.006 & 0.001 & 0.125 & 177.540 & 7.604 \\
\hline CLTC-P & 1.464 & 0.227 & 0.212 & 0.025 & 0.515 & 257.680 & 11.150 \\
\hline CLTC-P 1P & 8.089 & 1.314 & 1.232 & 0.117 & 2.257 & 480.450 & 21.271 \\
\hline CLTC-P 2P & 0.117 & 0.004 & 0.003 & 0.006 & 0.156 & 245.310 & 10.500 \\
\hline CLTC-P 3P & 0.086 & 0.003 & 0.002 & 0.006 & 0.157 & 179.680 & 7.691 \\
\hline
\end{tabular}


Table3 lists the combined emissions and fuel consumption values of the test vehicle under three test cycle conditions at each phase of a single cycle. First, a comparison of the combined emissions of gaseous pollutants under the three cycles is made as shown in Figure 4:

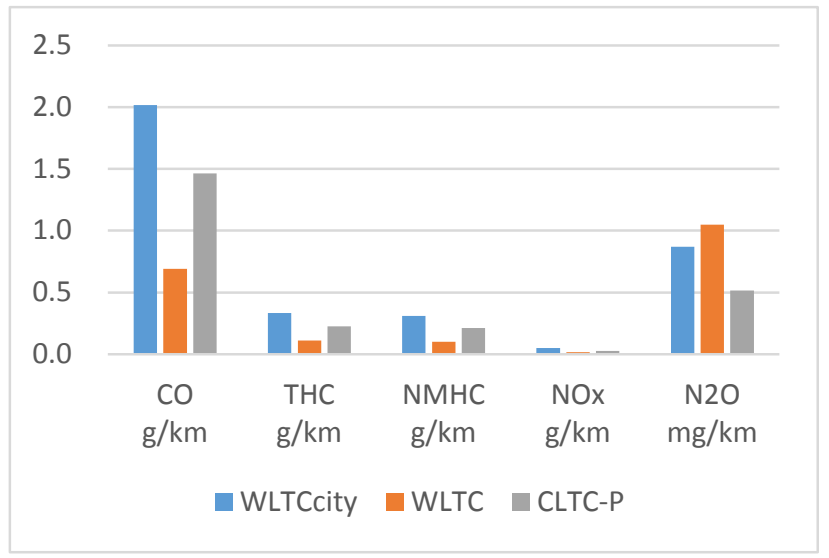

Fig. 4. Comparison of gaseous pollutants in three test cycles.

It can be seen from the figure that for carbon monoxide (CO), total hydrocarbons (THC), non-methane hydrocarbons (NMHC) and nitrogen oxides (NOx) The combined emissions of the WLTC city cycle are greater than the combined emissions of the WLTC cycle. The combined emissions under the CLTC-P cycle are approximately twice as high as those under the WLTC cycle. The combined emissions of the WLTC cycle are greater than those of the WLTC city cycle for the gaseous pollutant nitrous oxide $\left(\mathrm{N}_{2} \mathrm{O}\right)$ than those under the CLTC-P cycle The combined emissions under the CLTC-P cycle are about half of those under the WLTC cycle.

The emissions of four gaseous pollutants at each phase of the WLTC city and WLTC cycles were compared separately, as shown in figure 5.

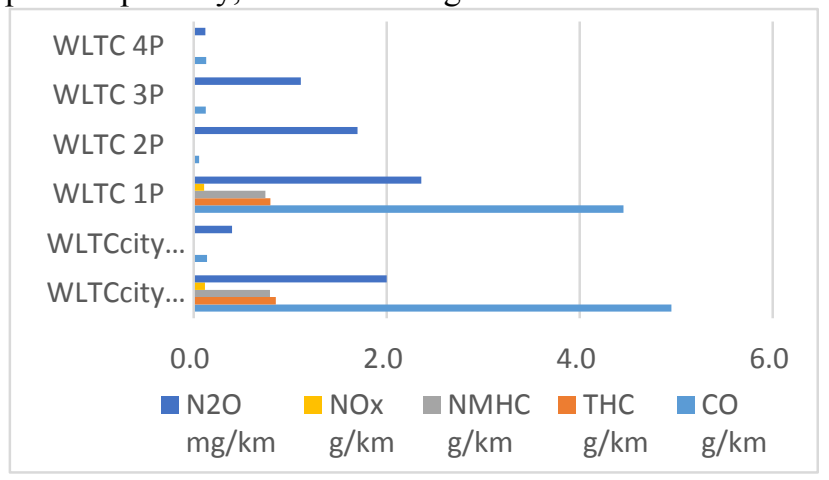

Fig. 5. Comparison of phase gas pollutants in WLTC city and WLTC cycles.

It can be seen from figure 5 that the emission of gaseous pollutants is mainly produced in the first phase, and the emission level of pollutants in the first phase under the two cycles is approximately the same. Not difficult to understand, because the WLTC city cycle is originally part of the WLTC cycle, the first phase of the two cycles is exactly the same working conditions, so the emission level of pollutants in the first phase is roughly the same. 
The emissions of four gaseous pollutants at each phase of the CLTC-P and WLTC cycles were compared separately, as shown in figures 6 and 7. It can be seen from the diagram that the emission of gaseous pollutants is mainly produced in the first phase, and the emission level of pollutants in the first phase of the two cycles is very different. In the first phase nitrous oxide $\left(\mathrm{N}_{2} \mathrm{O}\right)$ and nitrogen oxide (NOx) emission levels are basically the same. The emission levels of the three pollutants THC, CO and NMHC under the CLTC-P cycle are significantly higher than those in the WLTC cycle, which is about twice the combined emissions under the WLTC cycle.

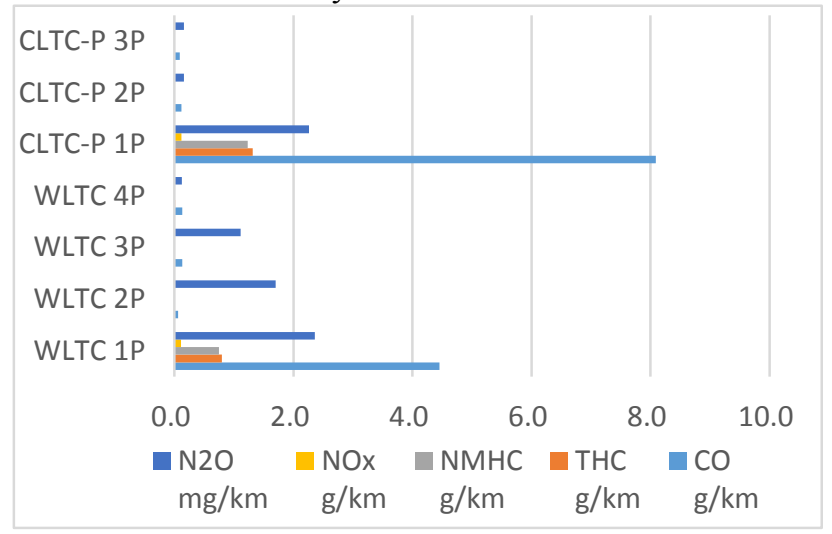

Fig. 6. Comparison of phase gas pollutants in WLTC and CLTC-P cycle.

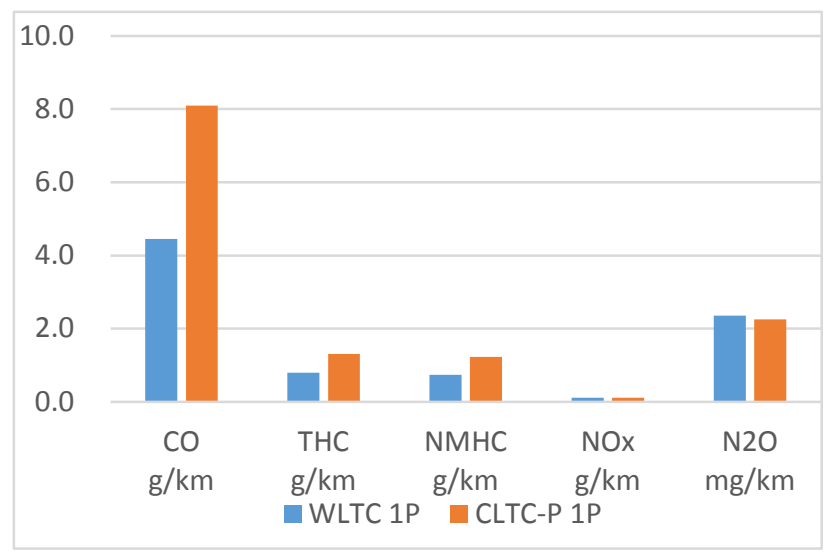

Fig. 7. Comparison of first-phase emissions in WLTC and CLTC-P cycle.

Then, the comparison of low temperature comprehensive fuel consumption under three cycles is carried out. The WLTC city cycle fuel consumption is the highest for the cycle comprehensive fuel consumption, as shown in figure 8 , is $11.73 \mathrm{~L} / 100 \mathrm{~km}$; The WLTC cycle fuel consumption is the lowest, $9.06 \mathrm{~L} / 100 \mathrm{~km}$; The CLTC-P cycle comprehensive fuel consumption is in the middle, $11.15 \mathrm{~L} / 100 \mathrm{~km}$. We can see that under the low temperature state, under the WLTC city working condition the comprehensive fuel consumption is about 1.3 times of the comprehensive fuel consumption under the complete WLTC cycle. Comprehensive fuel consumption under CLTC-P Working Conditions is about 1.25 times of the comprehensive fuel consumption under the complete WLTC cycle. 


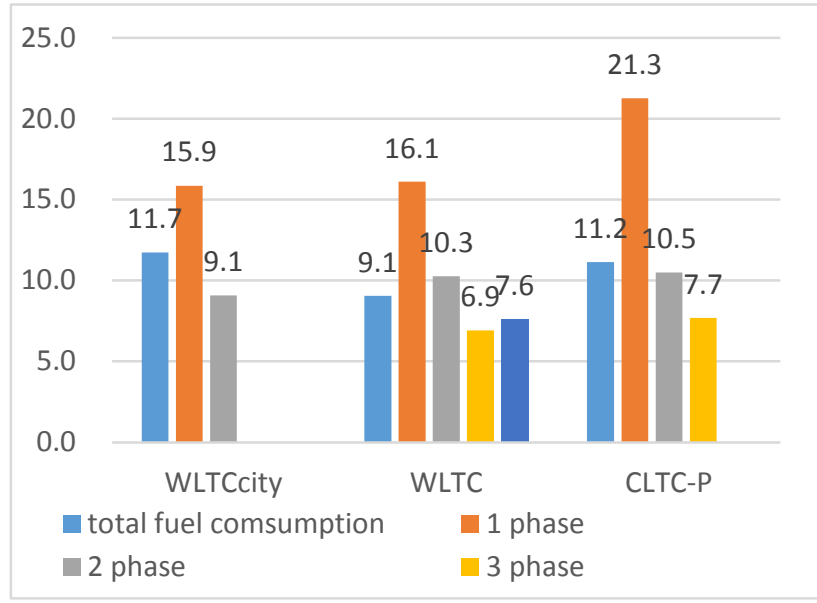

Fig. 8. Comparison of three cycles combined fuel consumption (L/100km).

It can also be found from figure 8 that the integrated fuel consumption in the first phase of each cycle condition is the highest, and the integrated fuel consumption in the first phase of CLTC-P cycle is $21.271 \mathrm{~L} / 100 \mathrm{~km}$, which is about 1.32 times that of the integrated fuel consumption $(16.101 \mathrm{~L} / 100 \mathrm{~km})$ in the first phase of WLTC cycle. The combined fuel consumption level of the second phase under the three cycles is approximately the same, and the combined fuel consumption level of the fourth phase of the WLTC cycle (extra-high speed section) and the third phase of the CLTC-P cycle (high speed section) is approximately the same.

The instantaneous emission curves of four gaseous pollutants $\mathrm{CO}, \mathrm{THC}, \mathrm{NOx}$ and $\mathrm{N}_{2} \mathrm{O}$ under WLTC and CLTC-P cycles are shown in Fig.9 and Fig.10, respectively, where the units of CO, THC and NOx are the units of $\mathrm{g} / \mathrm{s}, \mathrm{N}_{2} \mathrm{O}$ is the units of $\mathrm{mg} / \mathrm{s}$. It can be seen from the image that the emission peaks of gaseous pollutants in 4 appear within 50 seconds after the cold start of the first phase.

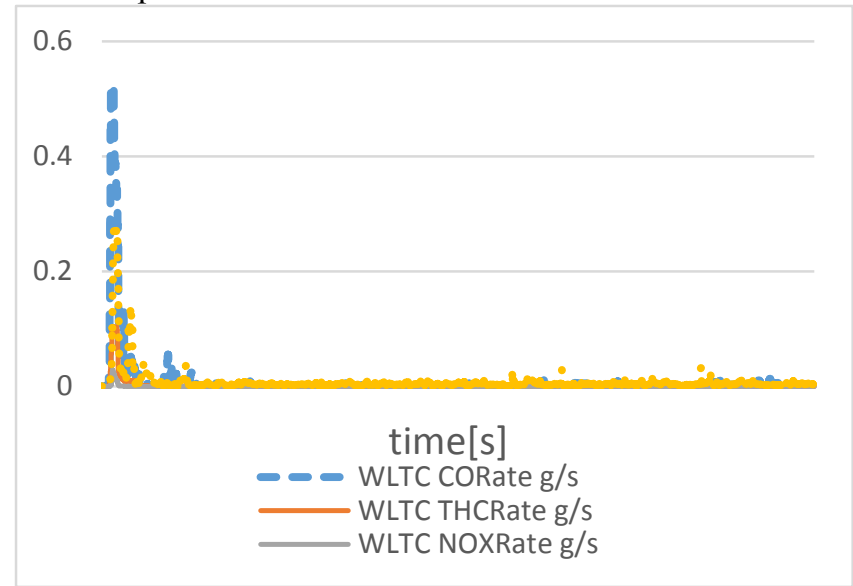

Fig. 9. Comparison of Transient Emissions of 4 Gaseous Pollutants per Second WLTC Cycle. 


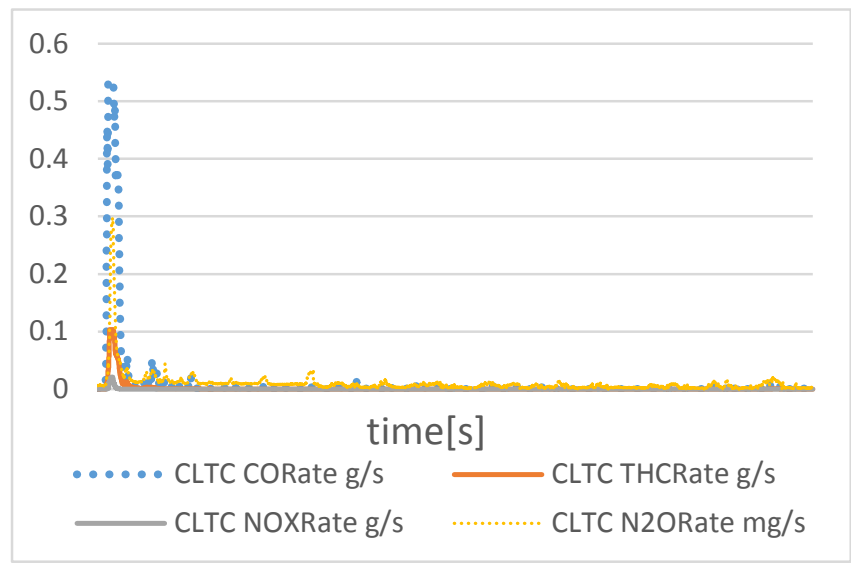

Fig. 10. Comparison of Transient Emissions of 4 Gaseous Pollutants per Second CLTC Cycle.

Draw the 1 st minute pollutant transient emissions is shown in figure 11-14. We can see that the peak of CO in the WLTC cycle appears at 25.4 seconds, the peak of CO emission is $0.52 \mathrm{~g} / \mathrm{s}$, the peak of concentration is $2597.49 \mathrm{ppm}$, the peak of CO in the CLTC-P cycle appears at 27.0 seconds, and the peak of $\mathrm{CO}$ emission is $0.53 \mathrm{~g} / \mathrm{s}$, the peak of concentration is $2618.36 \mathrm{ppm}$;THC peaks in in the WLTC cycle appears at 28.8 seconds, the peak of THC emission is $0.103 \mathrm{~g} / \mathrm{s}$, the peak of concentration is $1043.05 \mathrm{ppm}$; the peak of THC in the CLTC-P cycle appears at 30.9 seconds, and the peak of THC emission is $0.103 \mathrm{~g} / \mathrm{s}$, concentration peak is 1029.14 ppm; The peak of NOx in the WLTC cycle appeared at 28.7 seconds, NOx emission peak was $0.029 \mathrm{~g} / \mathrm{s}$, concentration peak was $113.98 \mathrm{ppm}$; the peak in the CLTC-P cycle appeared at 38.0 seconds, NOx emission peak was $0.021 \mathrm{~g} / \mathrm{s}$, concentration peak was 82.86 ppm; The peak of $\mathrm{N}_{2} \mathrm{O}$ in the WLTC cycle appears at 38.9 seconds, the peak of $\mathrm{N}_{2} \mathrm{O}$ emission is $0.275 \mathrm{mg} / \mathrm{s}$, the peak of concentration is $0.875 \mathrm{ppm}$; the peak of $\mathrm{N}_{2} \mathrm{O}$ in the CLTC-P cycle appears at 38.5 seconds, and the peak of $\mathrm{N}_{2} \mathrm{O}$ emission is $0.298 \mathrm{mg} / \mathrm{s}$, the peak of concentration is $0.938 \mathrm{ppm}$.

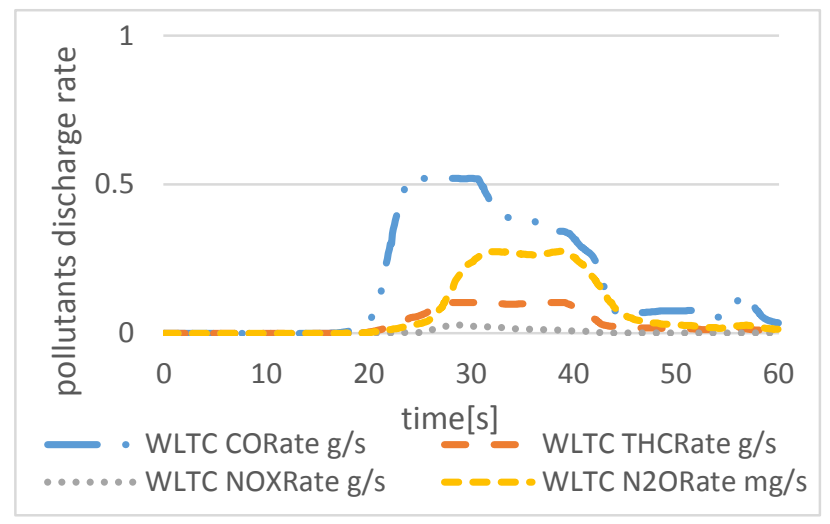

Fig. 11. WLTC Emission rates of circulating gaseous pollutants. 


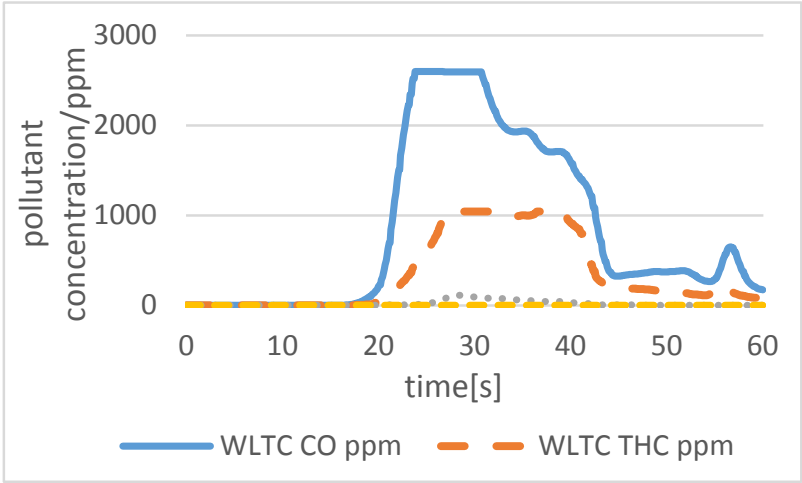

Fig. 12. WLTC Emission concentrations of circulating gaseous pollutants.

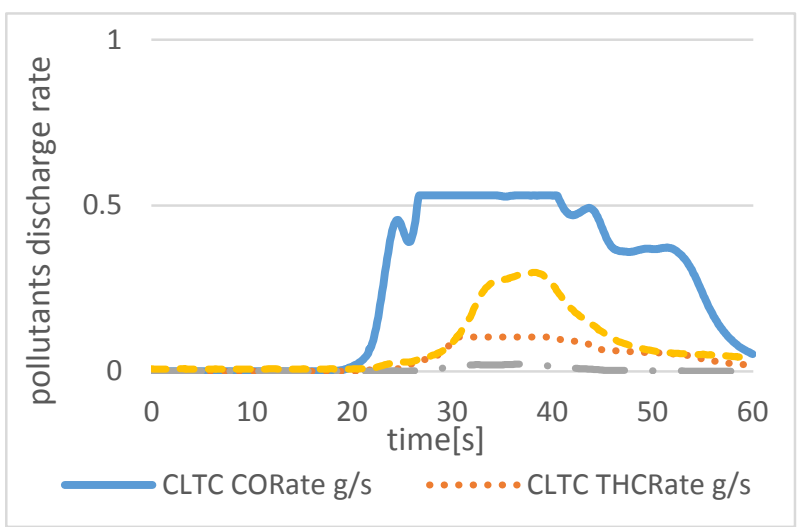

Fig. 13. CLTC-P Emission rates for circulating gaseous pollutants.

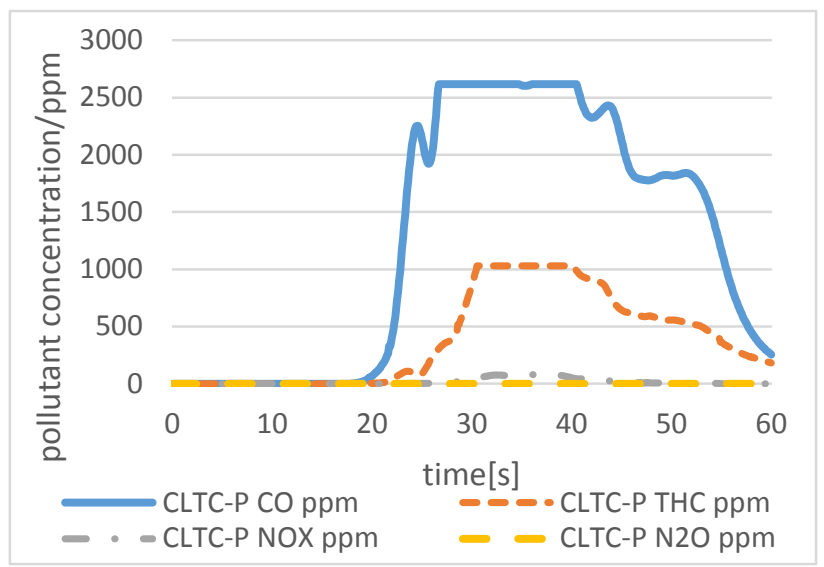

Fig. 14. CLTC-P Emission concentrations of circulating gaseous pollutants.

\section{Conclusions}

(1) For CO, THC, NMHC and NOx four pollutants, the combined WLTC city cycle emissions are the largest, the combined WLTC cycle emissions are the smallest, and the combined CLTC-P cycle emissions are the middle. The combined emissions under the 
CLTC-P cycle are approximately twice as high as those under the WLTC cycle. The combined emissions of WLTC cycle are the largest for $\mathrm{N}_{2} \mathrm{O}$, the smallest for CLTC-P cycle, and the intermediate for WLTC city cycle. The combined emissions under the CLTC-P cycle are about half that under the WLTC cycle.

(2) Emissions of gaseous pollutants are mainly generated in the first phase.The first phase except nitrous oxide (N2O) and nitrogen oxide (NOx) emission levels are basically the same, the emission levels of the three pollutants THC, CO and NMHC under the CLTC-P cycle are significantly higher than those in the WLTC cycle, which is about twice the combined emissions under the WLTC cycle.

(3) The combined fuel consumption under low temperature WLTC city working conditions is about 1.3 times that under the complete WLTC cycle. The comprehensive fuel consumption under CLTC-P working conditions is about 1.25 times that of the complete WLTC cycle. The combined fuel consumption level of the second phase under the three cycles is approximately the same, and the combined fuel consumption level of the fourth phase of the WLTC cycle (extra-high speed section) and the third phase of the CLTC-P cycle (high speed section) is approximately the same.

(4) For cold-start instantaneous emissions, except for NOx instantaneous emission peaks, the peak levels of emissions of $\mathrm{CO}$, THC and $\mathrm{N}_{2} \mathrm{O}$ gaseous pollutants are approximately the same, and the main factor that varies in the overall level of emissions is the duration of the pollutant emission peaks.

\section{Reference}

1. Bielaczyc, P., Woodburn, J., and Szczotka, A., "A Comparison of Carbon Dioxide Exhaust Emissions and Fuel Consumption for Vehicles Tested over the NEDC, FTP-75 and WLTC Chassis Dynamometer Test Cycles," SAE Technical Paper 2015-01-1065, 2015, doi:10.4271/2015-01-065.

2. Wang Jiaqing. Development trend and problems of world automobile in $21 \mathrm{st}$ century [J]. Volkswagen ,1998(1):38-40.(in Chinese).

3. Fu Guobin, Li Ke Jean. Advances in Global Warming and Wetland Ecosystems [J]. Geographic Studies ,2001,20(1):120-128.(in Chinese).

4. May, J., Bosteels, D., Favre, C., "A Comparison of Light-Duty Vehicle Emissions over Different Test Cycles and in Real Driving Conditions," FISITA paper F2014-CET-058, 2014.

5. Marotta, A., Pavlovic, J., Ciuffo, B., Serra, S., and Fontaras, G., "Gaseous Emissions from Light-Duty Vehicles: Moving from NEDC to the New WLTP Test Procedure," Environmental Science \& Technology 201549 (14), 8315-8322, doi:10.1021/acs.est.5b01364.

6. Wang Xia, Chu Guanyao, Yu Fei. The Influence of Resistance Loading on WLTC Cycle Emission and Fuel Consumption of Passenger Vehicles [J]. Transport Energy Conservation and Environmental Protection ,2019(03 2019):5-7.(in Chinese).

7. Simpson A. Cost -Benefit Analysis of Plug -In Hybrid Electric Vehicle Technology. The 22nd InternationalBattery, Hybrid, and Fuel Cell Electric Vehicle Symposium and Exposition, October 2006.

8. The Engineering Society for Advancing Mobility Land Sea Air and Space. Recommended Practice for Measuring FuelEconomy and Emissions of Hybrid Electric and Conventional Heavy-duty Vehicles. SAE J1711, 1999. 
9. The Engineering Society for Advancing Mobility Land Sea Air and Space. Recommended Practice for Measuring the Exhaust Emissions and Fuel Economy of Hybrid - Electric Vehicles. SAE J2711, 2002.

10. Qin Kongjian, Chen Haifeng, Fang Maodong, et al. A Study on the Evaluation Method of Emission and Energy Consumption of Plug-in Hybrid Electric Vehicle [J].. Automotive Technology ,2010,7(1).(in Chinese).

11. Yiwu Yang. A bright future for plug-in hybrid [J]. Cars and Accessories ,2012,2:21-23.(in Chinese).

12. Wang Shuqing, Cheng Chuanhui, Zhou Zhou, et al. Study on the Emission Characteristics of Particles Based on WLTC Test Cycle and Its Relation to Engine Working Conditions [J]. Shanghai Auto ,2019(10):5.(in Chinese).

13. AQSIQ. GB/T 38146.1-2019 China National Standard for Motor Driving Conditions Part 1: Light Vehicle [S]. 2019-10-18.(in Chinese).

14. Ministry of Environmental Protection. GB18352-2016 Light Vehicle Pollutant Emission Limits and Measurement Methods (China Stage 6)[S]. 2016-12-23.(in Chinese).

15. Society Automotive Engineers.J1711 -2010 Recommended Practice for Measuring the Exhaust Emissions and Fuel Economy of Hybrid -Electric Vehicles, Including Plug -in Hybrid Vehicles.USA:SAE,2010.

16. International Standard Organization. $23274-2007$ Hybrid -electric road vehicles-Exhaust emissions and fuel consumption measurements - Non -externally chargeable vehicles. UN:ISO,2007. 